\title{
PW02-021 - SAA1 is the strongest predictor of AA in TRAPS
}

\author{
L Obici ${ }^{1 *}$, HJ Lachmann², I Touitou ${ }^{3}$, G Palladini ${ }^{1}$, S Donadei ${ }^{4}$, M Pasotti ${ }^{1}$, L Cantarini ${ }^{5}$, K Gerhold $^{6}$, A Meini ${ }^{7}$, \\ N Toplak ${ }^{8}$, P Woo ${ }^{9}$, A Martini $^{10}$, G Merlini ${ }^{1}$, M Gattorno ${ }^{10}$ \\ From 7th Congress of International Society of Systemic Auto-Inflammatory Diseases (ISSAID) \\ Lausanne, Switerland. 22-26 May 2013
}

\section{Introduction}

AA amyloidosis is the most severe complication of hereditary autoinflammatory diseases. In TRAPS it has been reported to occur in approximately $25 \%$ of patients in the absence of an effective treatment. However, susceptibility to AA is difficult to predict. Identification of key risk factors affecting the development of AA would improve the clinical management of TRAPS patients, by allowing a more tailored treatment approach.

\section{Objectives}

To evaluate the relative contribution of clinical and genetic factors to the risk of AA amyloidosis in TRAPS.

\section{Methods}

Clinical data were obtained from the EUROFEVER/ EUROTRAPS web-based registry. Inclusion criteria for this study were: age $\geq 18$ years at last follow-up, identification of a TNFRSF1A mutation and written informed consent. DNA was available for patients recruited into the EUROTRAPS research project and SAA1 was genotyped by direct sequencing of exon 3 .

\section{Results}

104 patients (51 males, 49\%) with TRAPS (39 different mutations) were included in the study. Median age was 41 years (range 18-88), median age at TRAPS onset was 6 years (range $0.5-63$ ), and median age at diagnosis was 37 years (10-81). 21 patients had AA amyloidosis, with a median age at AA onset of 38 years (range 18-76). Family history for AA amyloidosis was observed in 33 patients (32\%). SAA1 genotype was established in 89/104 patients and 31 (35\%) were homozygous for the SAA1.1 allele.

'Amyloid Research and Treatment Centre, IRCCS Fondazione Policlinico San Matteo, Pavia, Italy

Full list of author information is available at the end of the article
77 patients (74\%) had a clearly pathogenic TNFRSF1A variant. 27 had either P46L or R92Q.

At univariate analysis, family history for amyloidosis, SAA1.1 homozygosity, disease course, age at TRAPS onset and the type of mutation were significantly associated with AA amyloidosis. At multivariate analysis homozygosity for SAA1.1 and age at TRAPS onset independently predicted development of renal amyloidosis. SAA1.1/1.1 genotype was the variable with the strongest influence on AA development, with a 5.3 fold increased risk whereas older age at TRAPS onset was associated with a reduced risk of AA amyloidosis. Survival according to SAA1 genotype (SAA1.1/SAA1.1 versus all other genotypes) was estimated by Kaplan-Meier analysis. Median amyloid free survival from birth was 47 years vs. not reached $(\mathrm{p}=0.01)$.

\section{Conclusion}

Homozygosity for the SAA1.1 allele is the strongest predictor of AA risk in TRAPS. This result is extremely relevant for the clinical management of TRAPS patients and supports $S A A 1$ genotyping on a routine basis to guide treatment approach.

\section{Disclosure of interest}

None declared.

\section{Authors' details}

'Amyloid Research and Treatment Centre, IRCCS Fondazione Policlinico San Matteo, Pavia, Italy. ${ }^{2}$ National Amyloidosis Centre, University College London Medical School, London, UK. ${ }^{3}$ INSERM U844, CHRU Montpellier, Montpellier, France. ${ }^{4}$ Laboratori Analisi Chimico Cliniche, IRCCS Fondazione Policlinico San Matteo, Pavia, Italy. ${ }^{5}$ Rheumatology Unit, Policlinico Le Scotte, Siena, Italy. ${ }^{6}$ Department of Pediatric Rheumatology, Charité Hospital, Berlin, Germany. ${ }^{7}$ Pediatric Immunology and Rheumatology, Spedali Civili, Brescia, Italy. ${ }^{8}$ University Children's Hospital, University Medical Centre Ljubljana, Ljubljana, Slovenia. ${ }^{9}$ Centre of Pediatric and Adolescent Rheumatology, UCL, London, UK. ${ }^{10}$ Giannina Gaslini Institute, Genova, Italy. 
- Convenient online submission

- Thorough peer review

- No space constraints or color figure charges

- Immediate publication on acceptance

- Inclusion in PubMed, CAS, Scopus and Google Scholar

- Research which is freely available for redistribution 